\title{
Growth Factors Released from Gelatin Hydrogel Microspheres Increase New Neurons in the Adult Mouse Brain
}

\author{
Kanako Nakaguchi, ${ }^{1}$ Hideo Jinnou, ${ }^{1,2}$ Naoko Kaneko, ${ }^{1}$ Masato Sawada, ${ }^{1}$ Takao Hikita, ${ }^{1}$ \\ Shinji Saitoh, ${ }^{2}$ Yasuhiko Tabata, ${ }^{3}$ and Kazunobu Sawamoto ${ }^{1}$ \\ ${ }^{1}$ Department of Developmental and Regenerative Biology, Nagoya City University Graduate School of Medical Sciences, \\ Aichi 467-8601, Nagoya, Japan \\ ${ }^{2}$ Department of Neonatology and Pediatrics, Nagoya City University Graduate School of Medical Sciences, \\ Aichi 467-8601, Nagoya, Japan \\ ${ }^{3}$ Department of Biomaterials, Institute for Frontier Medical Sciences, Kyoto University, Kyoto 606-8507, Japan
}

Correspondence should be addressed to Kazunobu Sawamoto, sawamoto@med.nagoya-cu.ac.jp

Received 23 June 2012; Accepted 9 August 2012

Academic Editor: Oscar Gonzalez-Perez

Copyright (C) 2012 Kanako Nakaguchi et al. This is an open access article distributed under the Creative Commons Attribution License, which permits unrestricted use, distribution, and reproduction in any medium, provided the original work is properly cited.

\begin{abstract}
Recent studies have shown that new neurons are continuously generated by endogenous neural stem cells in the subventricular zone (SVZ) of the adult mammalian brain. Some of these new neurons migrate to injured brain tissues and differentiate into mature neurons, suggesting that such new neurons may be able to replace neurons lost to degenerative disease or injury and improve or repair neurological deficits. Here, we tested whether delivering growth factors via gelatin hydrogel microspheres would support neurogenesis in the SVZ. Insulin-like growth factor-1 (IGF-1)-containing microspheres increased the number of new neurons in the SVZ. Hepatocyte growth factor (HGF)-containing microspheres increased the number of new neurons migrating from the SVZ towards the injured striatum in a stroke model in mouse. These results suggest that the strategy of using gelatin hydrogel microspheres to achieve the sustained release of growth factors holds promise for the clinical regeneration of damaged brain tissues from endogenous neural stem cells in the adult SVZ.
\end{abstract}

\section{Introduction}

Neural stem cells (NSCs) reside in the brain of adult animals, including humans [1-6]. NSCs residing in the subventricular zone (SVZ) at the lateral walls of the lateral ventricle and in the subgranular zone (SGZ) in the dentate gyrus of the hippocampus produce new neurons and glial cells throughout adult life in mammals [7-11]. The NSCs in the SVZ have the potential to regenerate lost neurons and glia in response to various pathological conditions [12-17]. Neuroregenerative therapy using endogenous NSCs in the SVZ is a highly anticipated emerging strategy for treating human brain diseases because it avoids the risk of immunological incompatibility and the ethical problems inherent in harvesting human cells, and it may reduce the risk of tumorigenesis-which are all problems associated with transplanted stem cells $[18,19]$. However, the spontaneous regeneration that takes place in the injured brain is insufficient for its structural or functional restoration $[12,17]$. For future clinical applications, the ability to regulate each step in neuronal regeneration, including the generation, migration, differentiation, survival, and functional maturation of new neurons to promote efficient regeneration will be crucial for developing novel and reliable neuronal self-repair strategies $[9,20-23]$.

Various proteins, including neurotrophic factors and paracrine signaling molecules, are reported to enhance neurogenesis in the SVZ [24]. However, one limitation of using these factors in the treatment of brain diseases is the lack of appropriate delivery systems. It is difficult to engineer systemically administered proteins to cross the blood-brain barrier, and such proteins can cause systemic toxicity at high concentrations $[25,26]$. On the other hand, a single local injection of liquid drugs into the brain parenchyma 
may not enhance neuronal regeneration effectively, given the limited volume and persistence of substances administered in this way. Therefore, for clinical applications, safe and effective methods for the sustained delivery of neurogenesisenhancing factors to the SVZ or injured neural tissues must be developed.

Here, we report that growth factors released from biodegradable gelatin hydrogel microspheres increase new neurons in the adult mouse brain. Gelatin hydrogels consist of gelatin polymers, which can be electrically complexed with growth factors [27, 28]. They have been used clinically to deliver growth factors in the treatment of patients with diseases including sudden sensorineural hearing loss, Bell's palsy, and peripheral artery diseases [29-32]. In this paper, we tested the effects of insulin-like growth factor (IGF) $[33,34]$ and hepatocyte growth factor (HGF) [35-37] delivered by gelatin hydrogel microspheres on neurogenesis in the SVZ in the normal brain, and then on the recruitment of SVZ-derived new neurons to the injured brain after stroke in a mouse model.

\section{Materials and Methods}

2.1. Preparation of Gelatin Hydrogel. Gelatin hydrogel microspheres (MedGel P15; MedGel, Osaka, Japan) with a diameter within $30 \mu \mathrm{m}$ were prepared as described previously $[27,28]$. The microspheres were incubated with phosphate buffered saline (PBS) (control) or PBS containing recombinant human IGF-1 (PeproTech, Rocky Hill, USA; $0.25 \mu \mathrm{g}$ ) or recombinant human HGF (PeproTech, Rocky Hill, USA; $0.5 \mu \mathrm{g}$ ) for 1 hour at room temperature.

2.2. Animals. Adult ( 8 weeks old) male ICR mice were purchased from SLC (Shizuoka, Japan) and maintained on a 12-hour light/dark cycle with unlimited access to food and water. All animal-related procedures were approved by the Laboratory Animal Care and Use Committee of Nagoya City University.

2.3. Injection of Gelatin Hydrogel. In intact mice, $3 \mu \mathrm{L}$ of the gelatin hydrogel suspension or $2 \mu \mathrm{L}$ of the solutions without gelatin hydrogel were stereotaxically injected using a capillary micropipette (Drummond Scientific Company, Broomall, PA, USA) into the striatum of anaesthetized 8-week-old ICR mice at the following position relative to bregma: $1.0 \mathrm{~mm}$ anterior, $1.5 \mathrm{~mm}$ lateral, and $3.2 \mathrm{~mm}$ deep $(n=5$ and 3 animals for groups treated with and without gelatin hydrogel, resp.). In the MCAO model, 11 days after MCAO, $5 \mu \mathrm{L}$ of gelatin hydrogel suspension or $2 \mu \mathrm{L}$ of the solutions without gelatin hydrogel were stereotaxically injected using a capillary micropipette into the striatum of anaesthetized 8-weekold ICR mice at the following position relative to bregma: $1.0 \mathrm{~mm}$ anterior, $2.0 \mathrm{~mm}$ lateral, and $3.2 \mathrm{~mm}$ deep $(n=6$ and 4 animals for groups treated with and without gelatin hydrogel, resp.). After surgery, the animals were left on a heat mat and constantly monitored until recovery. After 7 days, the mice were killed and their brains were prepared for immunohistochemistry.
2.4. Middle Cerebral Artery Occlusion. Middle cerebral artery occlusion (MCAO) was accomplished using the previously described intraluminal filament technique [17, 38, 39]. A laser-Doppler flowmeter probe (model ALF21; Advance, Tokyo, Japan) was attached to the surface of the skull to monitor the regional cerebral blood flow. A silicone-coated 8-0 filament was inserted into the internal carotid artery through an incision in the external carotid artery and then advanced to occlude the middle cerebral artery (MCA). The occlusion of the MCA was confirmed by observing a reduction in the value of laser-Doppler flowmetry of about $30 \%$. The filament was withdrawn 50-60 minutes later, and reperfusion was confirmed by laser-Doppler flowmetry, and the incision was then closed. Gelatin hydrogel microspheres were injected 11 days after MCAO. Seven days after the injection, the mice were killed and their brains were prepared for immunohistochemistry.

2.5. Immunohistochemistry and Quantification. Brains were perfusion-fixed with $4 \%$ paraformaldehyde, postfixed in the same fixative overnight, and $50 \mu \mathrm{m}$ sections were cut on a Vibratome sectioning system (VT1200S; Leica, Heidelberg, Germany) as described previously [17, 38, 39]. After three rinses in PBS, the sections were incubated for $40 \mathrm{~min}$ in blocking solution (PBS containing 10\% donkey serum and $0.2 \%$ Triton $\mathrm{X}-100$ ) and then overnight at $4^{\circ} \mathrm{C}$ with primary antibodies, which were diluted in the same solution. The next day, the sections were incubated for 2 hours at room temperature with biotinylated secondary antibodies $(1: 500)$ (Jackson, West Grove, PA, USA) or Alexa Fluor-conjugated secondary antibodies $(1: 500)$ (Invitrogen, Carlsbad, CA, USA), unless otherwise noted. Biotinylated antibodies were visualized using the Vectastain Elite ABC kit (Vector Laboratories, Burlingame, CA, USA) and DAB (diaminobenzidine tetrahydrochloride).

The primary antibodies (final dilution and source) used in this study were goat anti-doublecortin (anti-DCX) (1:100; Santa Cruz Biotechnology, Santa Cruz, CA, USA), rabbit anti-Ki67 (1:200; Novocastra, Newcastle, UK), and mouse anti-NeuN (1:100; Merck Millipore, Billerica, MA, USA).

For laser scanning microscopy, we used an LSM700 Microscope (Carl Zeiss, Oberkochen, Germany). For the quantification of immunolabeled cells, the images of stained cells were acquired using a fluorescence microscope, BX51 (Olympus, Tokyo, Japan), and a CCD camera, DP71 (Olympus). The actual number of DCX + cells was counted in three sections: the one containing the injection site and two sections taken $600 \mu \mathrm{m}$ and $1200 \mu \mathrm{m}$, respectively, anterior to the injection site. For analyses of the brain after MCAO, the section including the injection site and an additional 5 (DCX) and 2 (Ki67) anterior sections spaced $300 \mu \mathrm{m}$ apart were used. The total cell number was estimated by multiplying the sum of the counted cells by 12 and 6 , respectively.

2.6. Infarct Volume Evaluation. Brain sections prepared after MCAO (thickness, $50 \mu \mathrm{m}$ ) were immunostained for NeuN 


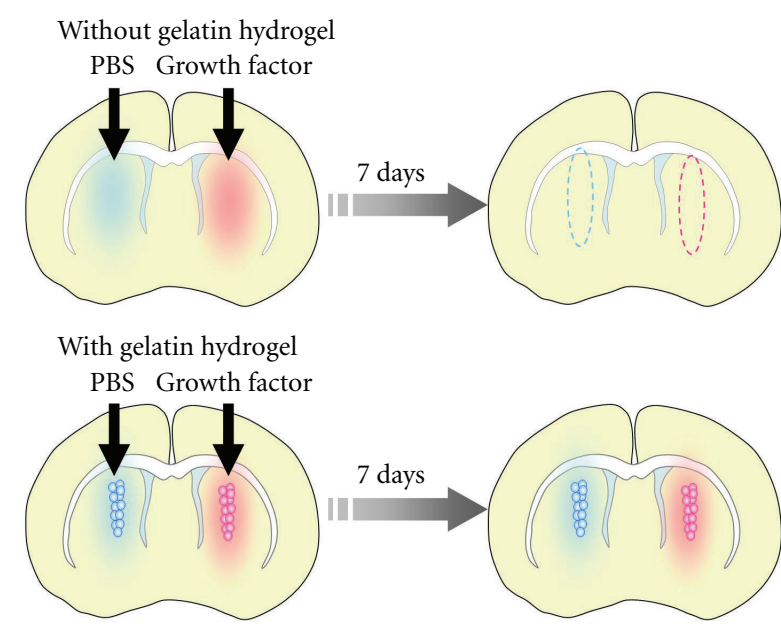

(a)

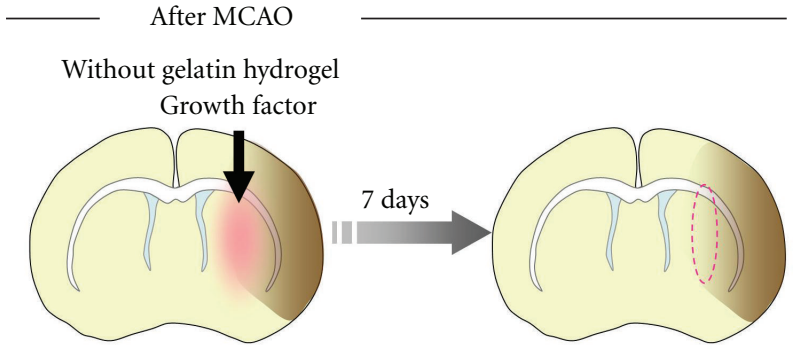

With gelatin hydrogel Growth factor

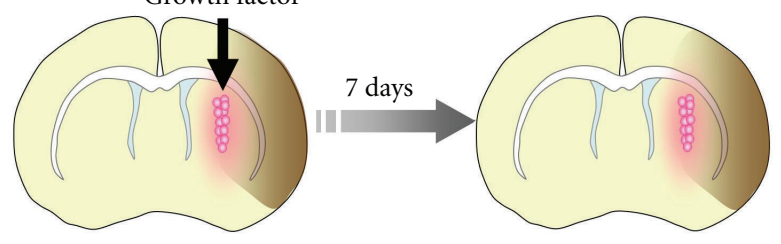

(b)

FIGURE 1: Study design. Schematic illustration of experiments performed to test the effects of growth factors released from gelatin hydrogel on neurogenesis in the SVZ. Effects of growth factors injected into normal (a) and injured brains 11 days after MCAO (b) with or without gelatin hydrogel on the number of new neurons in the SVZ and injured striatum, respectively, were compared 7 days after the injections. Blue and pink circles represent gelatin hydrogel microspheres.

and visualized with $\mathrm{DAB}$ using a standard procedure. The images of the sections including the injection site and two additional anterior sections spaced $600 \mu \mathrm{m}$ apart were captured using a fluorescence microscope, BX51 (Olympus, Tokyo, Japan), and a CCD camera, DP71 (Olympus).

For this analysis, we used only mice in which the striatal infarction (NeuN negative area) was observed within $1 \mathrm{~mm}$ of the SVZ in at least one section. The areas that lacked NeuN immunoreactivity (infarct area) and those of the ipsilateral hemisphere in the three sections were measured using the Photoshop CS 8.0.1 (Adobe Systems, San Jose, CA, USA) image software. The percentage of the infarct area was determined by dividing the infarct area by that of the ipsilateral hemisphere for each mouse and used for the analysis of infarct volume.

2.7. Statistics. All data are presented as the mean \pm SEM. Comparisons between experimental groups were analyzed by two-tailed Student's $t$-test, and differences were regarded as statistically significant when $P<0.05$.

\section{Results and Discussion}

3.1. Study Design. In this study, we focused on two growth factors, IGF-1 and HGF, incorporated into gelatin hydrogel, which can slowly release proteins after implantation into the body [27, 28]; both IGF-1 and HGF have been approved for clinical applications in the treatment of various diseases [29$32,40-42]$. The gelatin hydrogel microspheres were injected into the striatum of normal brain or close to the injured tissue of a stroke model (Figure 1). Seven days after the injection, the brains were fixed and number of DCX+ new neurons was compared.
3.2. IGF. IGF-1 promotes the proliferation of neural stem or progenitor cells as well as neuronal differentiation and survival [43-46]. It has a neuroprotective effect in animal models of stroke [47]. IGF-1-containing gelatin hydrogel microspheres have been used in a clinical trial for the treatment of patients with glucocorticoid-resistant sudden sensorineural hearing loss [29]. However, it is unknown whether the controlled release of IGF-1 using gelatin hydrogel is useful to stimulate neurogenesis in the SVZ.

We tested the effects of gelatin hydrogel containing IGF1 on the number of new neurons in the intact adult mouse SVZ. First, we injected PBS containing IGF-1 $(0.25 \mu \mathrm{g})$ without hydrogel into the striatum close to the SVZ (Figure 2(a)). Seven days later, there was no significant difference in the numbers of DCX+ new neurons in the SVZ between the IGF-1-injected and PBS-injected control brains (Figure 2(c), PBS: $1621 \pm 50$ cells, IGF-1: $1633 \pm 205$ cells, $n=3$ animals for each group), indicating that the IGF-1 injection without gelatin hydrogel was not effective in stimulating neurogenesis under these experimental conditions. We then injected a gelatin-hydrogel suspension in PBS, with or without the same amount of IGF-1, into the striatum (Figure 2(b)). Seven days later, significantly increased numbers of DCX+ new neurons were observed in the SVZ of brains that received the IGF-1-containing gelatin hydrogel compared with the control group (Figures 2(b) and 2(c), PBS: $1469 \pm 99$ cells, IGF-1: $1916 \pm 143$ cells, $n=5$ animals for each group). These results indicate that gelatin hydrogel is suitable as a vehicle for the delivery of IGF-1 to the SVZ. In addition, these results are consistent with the sustained release of IGF- 1 from gelatin hydrogel efficiently promoting IGF-1's actions on the proliferation and differentiation of neural stem or progenitor cells [43-46], resulting in increased numbers of new neurons in the SVZ. 


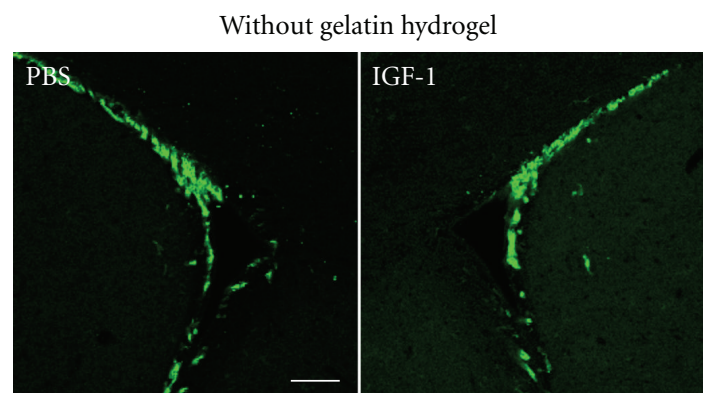

(a)

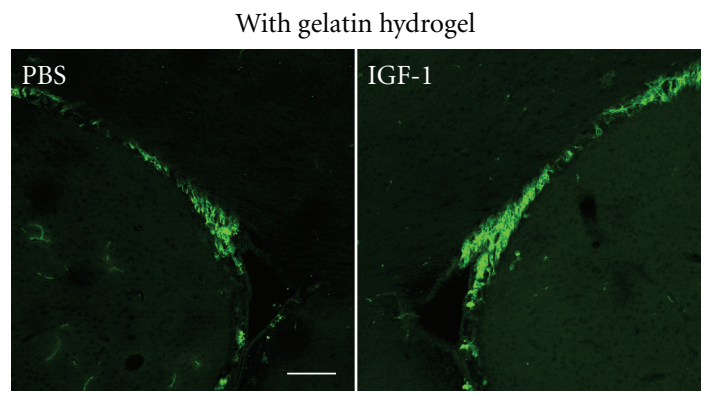

(b)

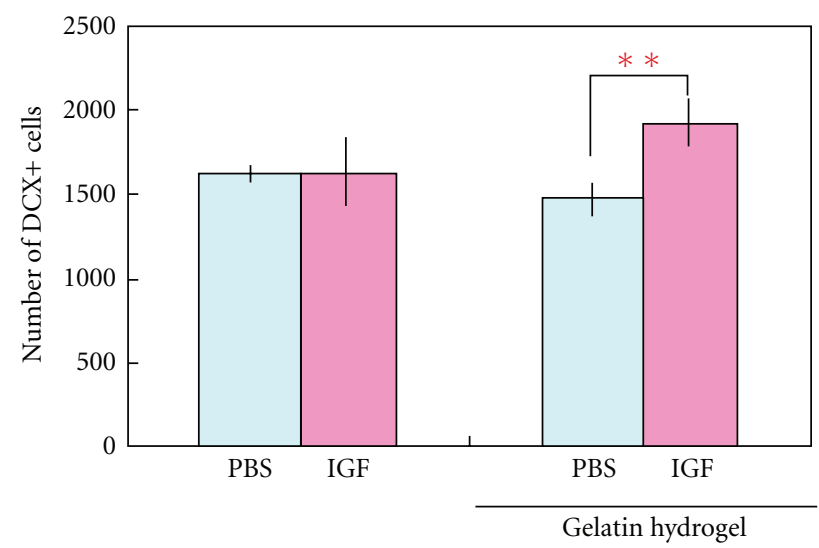

(c)

FIGURE 2: Effects of IGF-1-containing gelatin hydrogel on neurogenesis in the SVZ. (a) Coronal sections of brains that received an IGF-1 or PBS injection without gelatin hydrogel 7 days before, showing DCX+ new neurons in the SVZ (green) ( $n=3$ animals for each group). (b) Coronal sections of brains that received an injection of microspheres containing IGF-1 or microspheres plus PBS, showing DCX+ new neurons in the SVZ (green) ( $n=5$ animals for each group). (c) Quantification of DCX+ cells in the SVZ. Scale bars: $100 \mu \mathrm{m}(\mathrm{a}$ and b). ** $P<0.01$.

3.3. HGF. Previous studies indicated that HGF stimulates the proliferation of neural stem or progenitor cells in the adult SVZ, as well as the migration and differentiation of new neurons [48-52]. Acute injection of HGF into the adult striatum has a neuroprotective effect and promotes neurological recovery in a mouse model of stroke [53]. Topical application of HGF-containing gels on the surface of the cerebral cortex increases the number of new migrating neurons in the striatum of the MCAO model [54]. HGF-containing gelatin hydrogel has been used for the treatment of animal models of several diseases including noise-induced hearing loss [55] and collagen-induced arthritis [56]. These studies strongly suggest that HGF-containing gelatin hydrogel is likely to be useful to enhance neuronal regeneration after stroke.

We first tested the effects of HGF in PBS, with or without gelatin hydrogel, injected into the striatum, on the number of new neurons in the SVZ of the normal adult brain. Seven days after the injection, there was no significant difference in the number of DCX+ cells between the HGF and PBScontrol groups regardless of the use of gelatin hydrogel: with gelatin hydrogel, animals treated with PBS showed 1790 \pm 102 new cells, and those given HGF generated $1750 \pm 106$ cells ( $n=3$ animals for each group); without gelatin hydrogel, animals treated with PBS showed $2111 \pm 82$ cells; those treated with HGF generated $1992 \pm 78$ cells $(n=5$ animals for each group, $t$-test).

Next, we tested the effects of injecting the same substances in a mouse model of stroke induced by MCAO. In this model, SVZ-derived new neurons can be found migrating toward the injured striatum 2-3 weeks after the induction of ischemia [17]. Therefore, we injected HGF in PBS, with or without gelatin hydrogel, into the striatum 11 days after MCAO (Figures 3(a) and 3(b)). Eighteen days after MCAO, we quantified the infarct volume and number of new neurons in the striatum. The HGF administration did not affect the infarct volume, regardless of the use of gelatin hydrogel microspheres: with gelatin hydrogel, PBS yielded an infarct volume of $26.3 \pm 3.4 \%$, and those treated with HGF had an infarct volume of $25.3 \pm 2.0 \%$ ( $n=6$ animals for each group); without gelatin hydrogel, animals treated with PBS showed an infarct volume of $26.6 \pm 1.6 \%$ and those treated with HGF had an infarct volume of $29.6 \pm 3.6 \%$ ( $n=4$ animals, $t$-test $)$.

The administration of HGF without gelatin hydrogel also did not affect the number of DCX+ cells in the striatum (Figures 3(c) and 3(d)). However, the HGF-containing gelatin hydrogel significantly increased the number of DCX+ cells migrating in the striatum after MCAO (Figures 3(c) and $3(\mathrm{~d}))$. Interestingly, the majority of the DCX + cells extended 


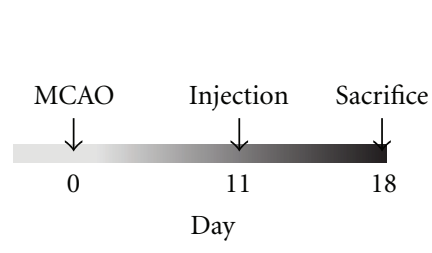

(a)

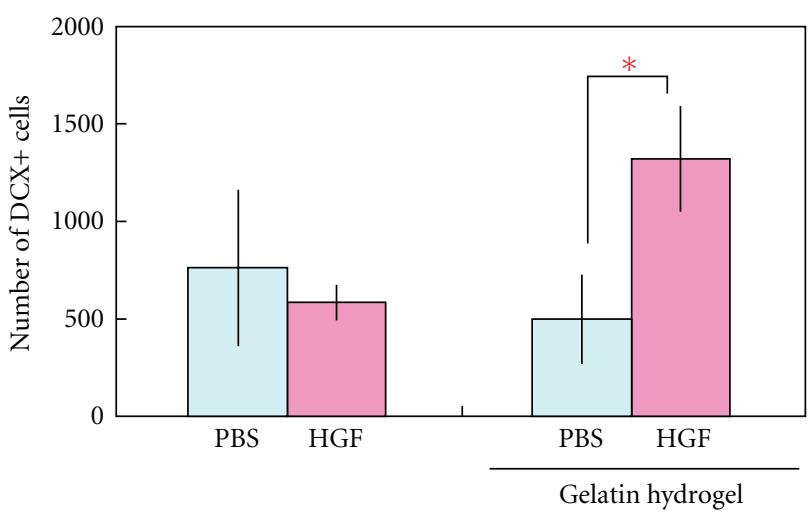

(d)

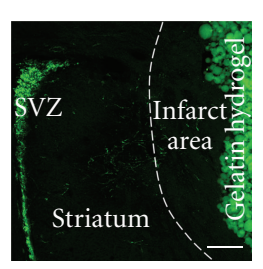

(b)

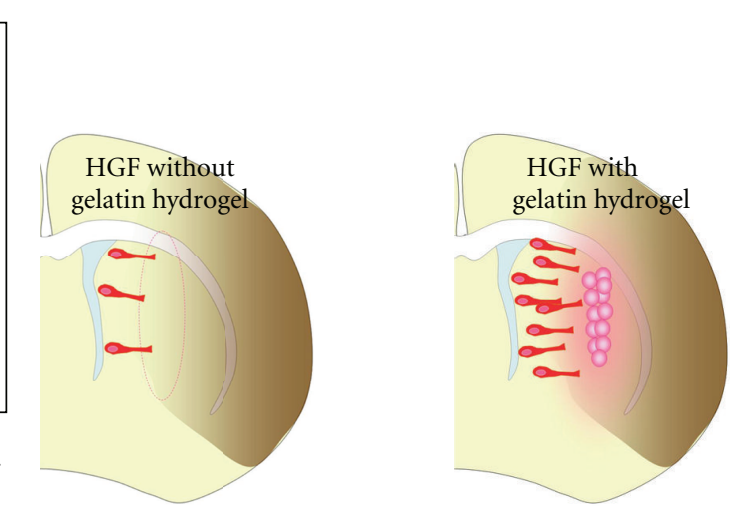

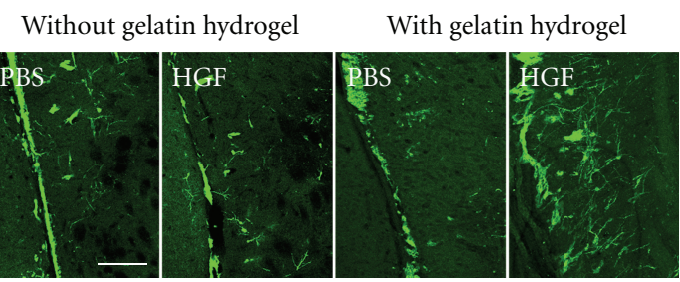

(c)

(e)

FIGURE 3: Effects of HGF-containing gelatin hydrogel on the number of new neurons migrating in the ischemic striatum after middle cerebral artery occlusion. (a) Experimental design. (b) A coronal brain section showing DCX+ new neurons in the SVZ (green) and gelatin hydrogel that was injected into the striatum. (c) Coronal sections stained for DCX (green) from brains that received a PBS or HGF injection with ( $n=6$ animals for each group) or without $(n=4$ animals for each group) gelatin hydrogel. (d) Quantification of the number of DCX+ cells separated by at least $50 \mu \mathrm{m}$ from the SVZ in the ipsilateral striatum. (e) Summary of the results. HGF administered as HGF-containing gelatin hydrogel microspheres significantly increased the number of new neurons. Scale bars: $200 \mu \mathrm{m}\left(\mathrm{b}\right.$ and c). ${ }^{*} P<0.05$.

a long leading process toward the injected gelatin hydrogel, suggesting that these cells were migrating laterally. Because HGF has been reported to stimulate the proliferation of SVZ progenitors [48], we also examined the effects of HGF on cell proliferation in the SVZ after MCAO. There was no significant difference in the number of Ki67+ proliferating cells between the HGF and PBS-control groups, regardless of the use of gelatin hydrogel (with gelatin hydrogel, PBS: $796.5 \pm 87.65$ cells, HGF: $798.8 \pm 96.12$ cells, $n=6$ and 5 animals, respectively; without gelatin hydrogel, PBS: $837.5 \pm$ 78.77 cells, HGF: $926.0 \pm 109.4$ cells, $n=4$ animals for each group, $t$-test). These results suggest that the increased number of new neurons in the injured striatum induced by treatment with HGF with gelatin hydrogel was due to an increased efficiency of the new neurons' migration but not to their production in the SVZ (Figure 3(e)).

Since the receptor for HGF, c-Met, is expressed in adult DCX+ migrating neurons [49] as well as GFAP+ astrocytes [50], it is possible that DCX+ cells are attracted by the HGF-releasing gelatin hydrogel. It is also possible that the HGF-induced increase in the number of DCX+ cells in the striatum was caused by angiogenic activity [54], since new neurons use blood vessels for their migration $[17,38]$.

\section{Conclusions}

The benefits reported here of using gelatin hydrogel microspheres to deliver growth factors to simulate neurogenesis in the SVZ demonstrate that gelatin hydrogel is a promising vehicle for the local and sustained release of drugs in the brain. Previous studies demonstrated that the epicortical delivery of EPO [57] and HGF [54] enhance neurogenesis in the SVZ in mouse models of stroke. However, considering the longer distance between the brain surface and the SVZ in the human brain, direct injection inside the brain parenchyma may be needed to efficiently stimulate neurogenesis from neural stem cells in the SVZ. The local delivery of drugs using hydrogels should result in a lower total dose of medications and thus in fewer side effects. In addition, this technology should be applicable to any charged protein that can enhance neural regeneration [58]. Further studies are needed to improve the injectability of the gelatin hydrogel, which will result in a less invasive procedure. Finally, how the newly generated neurons produced by this treatment contribute to neurological improvement needs to be elucidated.

\section{Authors' Contribution}

K. Nakaguchi and H. Jinnou contributed equally to this work.

\section{Acknowledgments}

The authors are grateful to Hiroshi Funakoshi (Asahikawa Medical University) and members of the Sawamoto 
Laboratory for discussions. M. Sawada is a Research Fellow of the Japan Society for the Promotion of Science. This work was supported by the Project for Realization of Regenerative Medicine (Ministry of Education, Culture, Sports, Science and Technology, Japan) and the Funding Program for Next Generation World-Leading Researchers (Japan Society for the Promotion of Science).

\section{References}

[1] A. Bédard and A. Parent, "Evidence of newly generated neurons in the human olfactory bulb," Developmental Brain Research, vol. 151, no. 1-2, pp. 159-168, 2004.

[2] P. S. Eriksson, E. Perfilieva, T. Björk-Eriksson et al., "Neurogenesis in the adult human hippocampus," Nature Medicine, vol. 4, no. 11, pp. 1313-1317, 1998.

[3] B. Kirschenbaum, M. Nedergaard, A. Preuss, K. Barami, R. A. R. Fraser, and S. A. Goldman, "In vitro neuronal production and differentiation by precursor cells derived from the adult human forebrain," Cerebral Cortex, vol. 4, no. 6, pp. 576-589, 1994.

[4] A. Quiñones-Hinojosa, N. Sanai, M. Soriano-Navarro et al., "Cellular composition and cytoarchitecture of the adult human subventricular zone: a niche of neural stem cells," Journal of Comparative Neurology, vol. 494, no. 3, pp. 415-434, 2006.

[5] N. S. Roy, S. Wang, L. Jiang et al., "In vitro neurogenesis by progenitor cells isolated from the adult human hippocampus," Nature Medicine, vol. 6, no. 3, pp. 271-277, 2000.

[6] H. Sanai, A. D. Tramontin, A. Quiñones-Hinojosa et al., "Unique astrocyte ribbon in adult human brain contains neural stem cells but lacks chain migration," Nature, vol. 427, no. 6976, pp. 740-744, 2004.

[7] A. Alvarez-Buylla and J. M. García-Verdugo, "Neurogenesis in adult subventricular zone," Journal of Neuroscience, vol. 22, no. 3, pp. 629-634, 2002.

[8] R. A. Ihrie and A. Álvarez-Buylla, "Lake-front property: a unique germinal niche by the lateral ventricles of the adult brain," Neuron, vol. 70, no. 4, pp. 674-686, 2011.

[9] N. Kaneko and K. Sawamoto, "Adult neurogenesis and its alteration under pathological conditions," Neuroscience Research, vol. 63, no. 3, pp. 155-164, 2009.

[10] P. M. Lledo, M. Alonso, and M. S. Grubb, "Adult neurogenesis and functional plasticity in neuronal circuits," Nature Reviews Neuroscience, vol. 7, no. 3, pp. 179-193, 2006.

[11] C. Zhao, W. Deng, and F. H. Gage, "Mechanisms and functional implications of adult neurogenesis," Cell, vol. 132, no. 4, pp. 645-660, 2008.

[12] A. Arvidsson, T. Collin, D. Kirik, Z. Kokaia, and O. Lindvall, "Neuronal replacement from endogenous precursors in the adult brain after stroke," Nature Medicine, vol. 8, no. 9, pp. 963-970, 2002.

[13] S. W. Levison and J. E. Goldman, "Both oligodendrocytes and astrocytes develop from progenitors in the subventricular zone of postnatal rat forebrain," Neuron, vol. 10, no. 2, pp. 201-212, 1993.

[14] B. Menn, J. M. Garcia-Verdugo, C. Yaschine, O. GonzalezPerez, D. Rowitch, and A. Alvarez-Buylla, "Origin of oligodendrocytes in the subventricular zone of the adult brain," Journal of Neuroscience, vol. 26, no. 30, pp. 7907-7918, 2006.

[15] J. M. Parent, Z. S. Vexler, C. Gong, N. Derugin, and D. M. Ferriero, "Rat forebrain neurogenesis and striatal neuron replacement after focal stroke," Annals of Neurology, vol. 52, no. 6, pp. 802-813, 2002.

[16] N. Picard-Riera, L. Decker, C. Delarasse et al., "Experimental autoimmune encephalomyelitis mobilizes neural progenitors from the subventricular zone to undergo oligodendrogenesis in adult mice," Proceedings of the National Academy of Sciences of the United States of America, vol. 99, no. 20, pp. 1321113216, 2002.

[17] T. Yamashita, M. Ninomiya, P. H. Acosta et al., "Subventricular zone-derived neuroblasts migrate and differentiate into mature neurons in the post-stroke adult striatum," Journal of Neuroscience, vol. 26, no. 24, pp. 6627-6636, 2006.

[18] L. Grabel, "Prospects for pluripotent stem cell therapies: into the clinic and back to the bench," Journal of Cellular Biochemistry, vol. 113, no. 2, pp. 381-387, 2012.

[19] K. Pfannkuche, T. Hannes, M. Khalil et al., "Induced pluripotent stem cells: a new approach for physiological research," Cellular Physiology and Biochemistry, vol. 26, no. 2, pp. 105$124,2010$.

[20] N. Kaneko, E. Kako, and K. Sawamoto, "Prospects and limitations of using endogenous neural stem cells for brain regeneration," Genes, vol. 2, no. 1, pp. 107-130, 2011.

[21] K. Nakaguchi, H. Masuda, N. Kaneko, and K. Sawamoto, "Strategies for regenerating striatal neurons in the adult brain by using endogenous neural stem cells," Neurology Research International, vol. 2011, Article ID 898012, 10 pages, 2011.

[22] H. Okano, M. Sakaguchi, K. Ohki, N. Suzuki, and K. Sawamoto, "Regeneration of the central nervous system using endogenous repair mechanisms," Journal of Neurochemistry, vol. 102, no. 5, pp. 1459-1465, 2007.

[23] H. Okano and K. Sawamoto, "Neural stem cells: Involvement in adult neurogenesis and CNS repair," Philosophical Transactions of the Royal Society B, vol. 363, no. 1500, pp. 2111-2122, 2008.

[24] G. Kempermann, "Regulation," in Adult Neurogenesis: Stem Cells and Neuronal Development in the Adult Brain, pp. 255325, Oxford University Press, New York, NY, USA, 2006.

[25] W. Löscher and H. Potschka, "Role of drug efflux transporters in the brain for drug disposition and treatment of brain diseases," Progress in Neurobiology, vol. 76, no. 1, pp. 22-76, 2005.

[26] A. Misra, S. Ganesh, A. Shahiwala, and S. P. Shah, "Drug delivery to the central nervous system: a review," Journal of Pharmacy and Pharmaceutical Sciences, vol. 6, no. 2, pp. 252273, 2003.

[27] Y. Tabata and Y. Ikada, "Protein release from gelatin matrices," Advanced Drug Delivery Reviews, vol. 31, no. 3, pp. 287-301, 1998.

[28] Y. Tabata and Y. Ikada, "Vascularization effect of basic fibroblast growth factor released from gelatin hydrogels with different biodegradabilities," Biomaterials, vol. 20, no. 22, pp. 2169-2175, 1999.

[29] T. Nakagawa, T. Sakamoto, H. Hiraumi et al., "Topical insulinlike growth factor 1 treatment using gelatin hydrogels for glucocorticoid-resistant sudden sensorineural hearing loss: a prospective clinical trial," BMC Medicine, vol. 8, article 76, 2010.

[30] A. Marui, Y. Tabata, S. Kojima et al., "A novel approach to therapeutic angiogenesis for patients with critical limb ischemia by sustained release of basic fibroblast growth factor using biodegradable gelatin hydrogel —an initial report of the phase I-IIa study," Circulation Journal, vol. 71, no. 8, pp. 11811186, 2007. 
[31] N. Hato, J. Nota, H. Komobuchi et al., "Facial nerve decompression surgery using bFGF-impregnated biodegradable gelatin hydrogel in patients with Bell palsy," Otolaryngology, vol. 146, no. 4, pp. 641-646, 2012.

[32] G. Takagi, M. Miyamoto, S. Tara et al., "Controlled-release basic fibroblast growth factor for peripheral artery disease: comparison with autologous bone marrow-derived stem cell transfer," Tissue Engineering A, vol. 17, no. 21-22, pp. 27872794, 2011.

[33] E. Rinderknecht and R. E. Humbel, "Amino terminal sequences of two polypeptides from human serum with nonsuppressible insulin like and cell growth promoting activities: evidence for structural homology with insulin B chain," Proceedings of the National Academy of Sciences of the United States of America, vol. 73, no. 12, pp. 4379-4381, 1976.

[34] E. Rinderknecht and R. E. Humbel, "The amino acid sequence of human insulin-like growth factor I and its structural homology with proinsulin," Journal of Biological Chemistry, vol. 253, no. 8, pp. 2769-2776, 1978.

[35] H. Funakoshi and T. Nakamura, "Hepatocyte growth factor: from diagnosis to clinical applications," Clinica Chimica Acta, vol. 327, no. 1-2, pp. 1-23, 2003.

[36] T. Nakamura, K. Nawa, and A. Ichihara, "Partial formation and characterization of hepatocyte growth factor from serum of hepatectomized rats," Biochemical and Biophysical Research Communications, vol. 122, no. 3, pp. 1450-1459, 1984.

[37] T. Nakamura, T. Nishizawa, M. Hagiya et al., "Molecular cloning and expression of human hepatocyte growth factor," Nature, vol. 342, no. 6248, pp. 440-443, 1989.

[38] T. Kojima, Y. Hirota, M. Ema et al., "Subventricular zonederived neural progenitor cells migrate along a blood vessel scaffold toward the post-stroke striatum," Stem Cells, vol. 28, no. 3, pp. 545-554, 2010.

[39] M. Ninomiya, T. Yamashita, N. Araki, H. Okano, and K. Sawamoto, "Enhanced neurogenesis in the ischemic striatum following EGF-induced expansion of transit-amplifying cells in the subventricular zone," Neuroscience Letters, vol. 403, no. 1-2, pp. 63-67, 2006.

[40] Y. Gu, J. Zhang, L. Guo et al., "A phase I clinical study of naked DNA expressing two isoforms of hepatocyte growth factor to treat patients with critical limb ischemia," The Journal of Gene Medicine, vol. 13, no. 11, pp. 602-610, 2011.

[41] T. D. Henry, A. T. Hirsch, J. Goldman et al., "Safety of a nonviral plasmid-encoding dual isoforms of heypatocyte growth factor in critical limb ischemia patients: a phase I study," Gene Therapy, vol. 18, no. 8, pp. 788-794, 2011.

[42] H. Shigematsu, K. Yasuda, T. Iwai et al., "Randomized, doubleblind, placebo-controlled clinical trial of hepatocyte growth factor plasmid for critical limb ischemia," Gene Therapy, vol. 17, no. 9, pp. 1152-1161, 2010.

[43] C. Vicario-Abejón, M. J. Yusta-Boyo, C. Fernández-Moreno, and F. De Pablo, "Locally born olfactory bulb stem cells proliferate in response to insulin-related factors and require endogenous insulin-like growth factor-I for differentiation into neurons and glia," Journal of Neuroscience, vol. 23, no. 3, pp. 895-906, 2003.

[44] G. J. Brooker, M. Kalloniatis, V. C. Russo et al., "Endogenous IGF-1 regulates the neuronal differentiation of adult stem cells," Journal of Neuroscience Research, vol. 59, no. 3, pp. 332$341,2000$.

[45] Y. Arsenijevic, S. Weiss, B. Schneider, and P. Aebischer, "Insulin-like growth factor-1 is necessary for neural stem cell proliferation and demonstrates distinct actions of epidermal growth factor and fibroblast growth factor-2," Journal of Neuroscience, vol. 21, no. 18, pp. 7194-7202, 2001.

[46] R. D. Hodge, A. Joseph D'Ercole, and J. R. O’Kusky, “Insulinlike growth factor-I accelerates the cell cycle by decreasing G1 phase length and increases cell cycle reentry in the embryonic cerebral cortex," Journal of Neuroscience, vol. 24, no. 45, pp. 10201-10210, 2004.

[47] R. Kooijman, S. Sarre, Y. Michotte, and J. D. Keyser, "Insulinlike growth factor I: a potential neuroprotective compound for the treatment of acute ischemic stroke?" Stroke, vol. 40, no. 4, pp. e83-e88, 2009.

[48] D. Garzotto, P. Giacobini, T. Crepaldi, A. Fasolo, and S. De Marchis, "Hepatocyte growth factor regulates migration of olfactory interneuron precursors in the rostral migratory stream through Met-Grb2 coupling," Journal of Neuroscience, vol. 28, no. 23, pp. 5901-5909, 2008.

[49] C. Nicoleu, O. Benzakour, F. Agasse et al., "Endogenous hepatocyte growth factor is a niche signal for subventricular zone neural stem cell amplification and self-renewal," Stem Cells, vol. 27, no. 2, pp. 408-419, 2009.

[50] T. W. Wang, H. Zhang, M. R. Gyetko, and J. M. Parent, "Hepatocyte growth factor acts as a mitogen and chemoattractant for postnatal subventricular zone-olfactory bulb neurogenesis," Molecular and Cellular Neuroscience, vol. 48, no. 1, pp. 38-50, 2011.

[51] J. Kokuzawa, S. Yoshimura, H. Kitajima et al., "Hepatocyte growth factor promotes proliferation and neuronal differentiation of neural stem cells from mouse embryos," Molecular and Cellular Neuroscience, vol. 24, no. 1, pp. 190-197, 2003.

[52] M. Kato, S. Yoshimura, J. Kokuzawa et al., "Hepatocyte growth factor promotes neuronal differentiation of neural stem cells derived from embryonic stem cells," NeuroReport, vol. 15, no. 1, pp. 5-8, 2004.

[53] T. R. Doeppner, B. Kaltwasser, A. Elali, A. Zechariah, D. M. Hermann, and M. Bähr, "Acute hepatocyte growth factor treatment induces long-term neuroprotection and stroke recovery via mechanisms involving neural precursor cell proliferation and differentiation," Journal of Cerebral Blood Flow and Metabolism, vol. 31, no. 5, pp. 1251-1262, 2011.

[54] J. Shang, K. Deguchi, Y. Ohta et al., "Strong neurogenesis, angiogenesis, synaptogenesis, and antifibrosis of hepatocyte growth factor in rats brain after transient middle cerebral artery occlusion," Journal of Neuroscience Research, vol. 89, no. 1, pp. 86-95, 2011.

[55] T. Inaoka, T. Nakagawa, Y. S. Kikkawa et al., "Local application of hepatocyte growth factor using gelatin hydrogels attenuates noise-induced hearing loss in guinea pigs," Acta OtoLaryngologica, vol. 129, no. 4, pp. 453-457, 2009.

[56] K. Okunishi, O. Sasaki, T. Okasora et al., "Intratracheal delivery of hepatocyte growth factor directly attenuates allergic airway inflammation in mice," International Archives of Allergy and Immunology, vol. 149, supplement 1, pp. 14-20, 2009.

[57] Y. Wang, M. J. Cooke, C. M. Morshead, and M. S. Shoichet, "Hydrogel delivery of erythropoietin to the brain for endogenous stem cell stimulation after stroke injury," Biomaterials, vol. 33, no. 9, pp. 2681-2692, 2012.

[58] R. J. Dempsey and H. S. G. Kalluri, "Ischemia-induced neurogenesis: role of growth factors," Neurosurgery Clinics of North America, vol. 18, no. 1, pp. 183-190, 2007. 

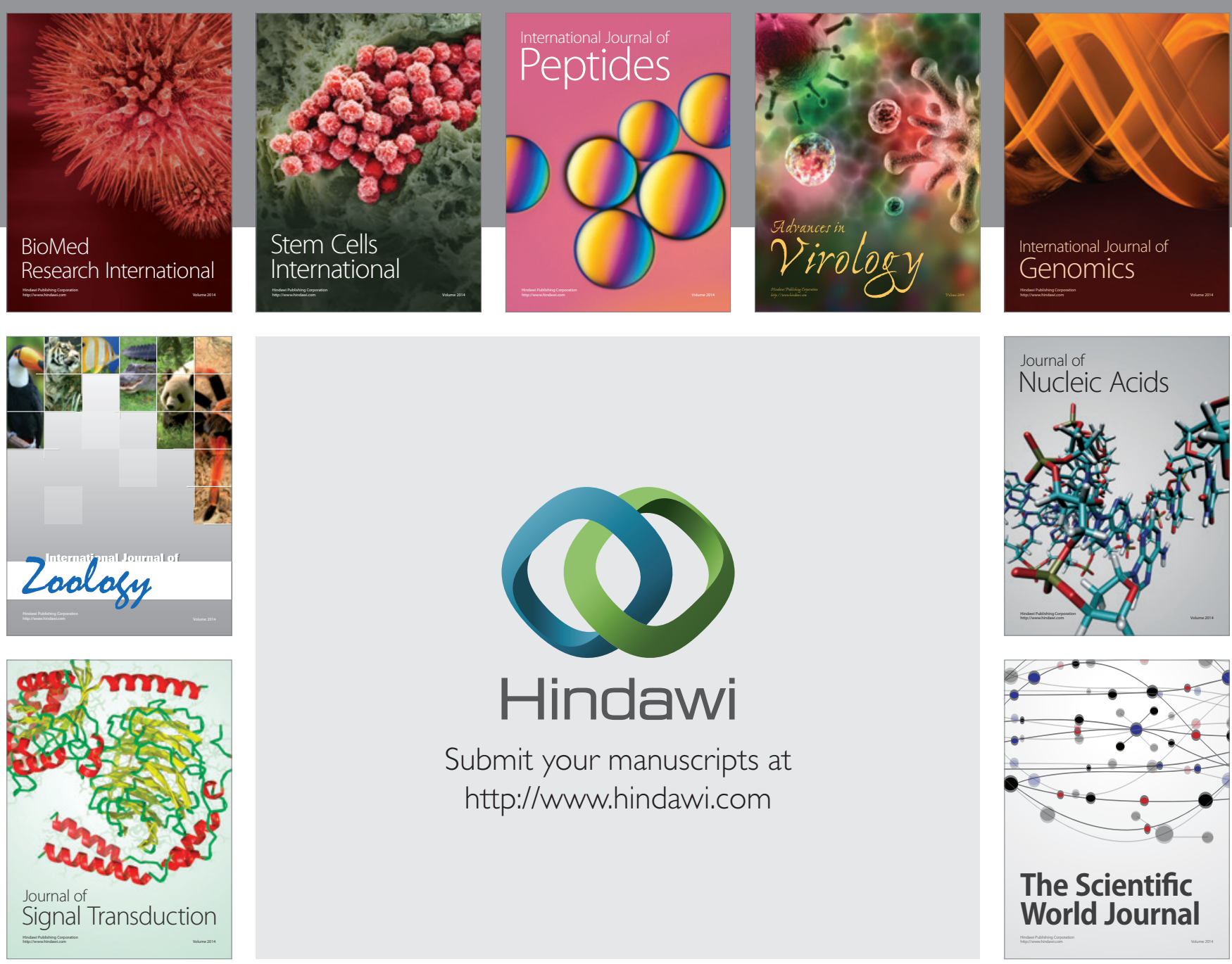

Submit your manuscripts at

http://www.hindawi.com
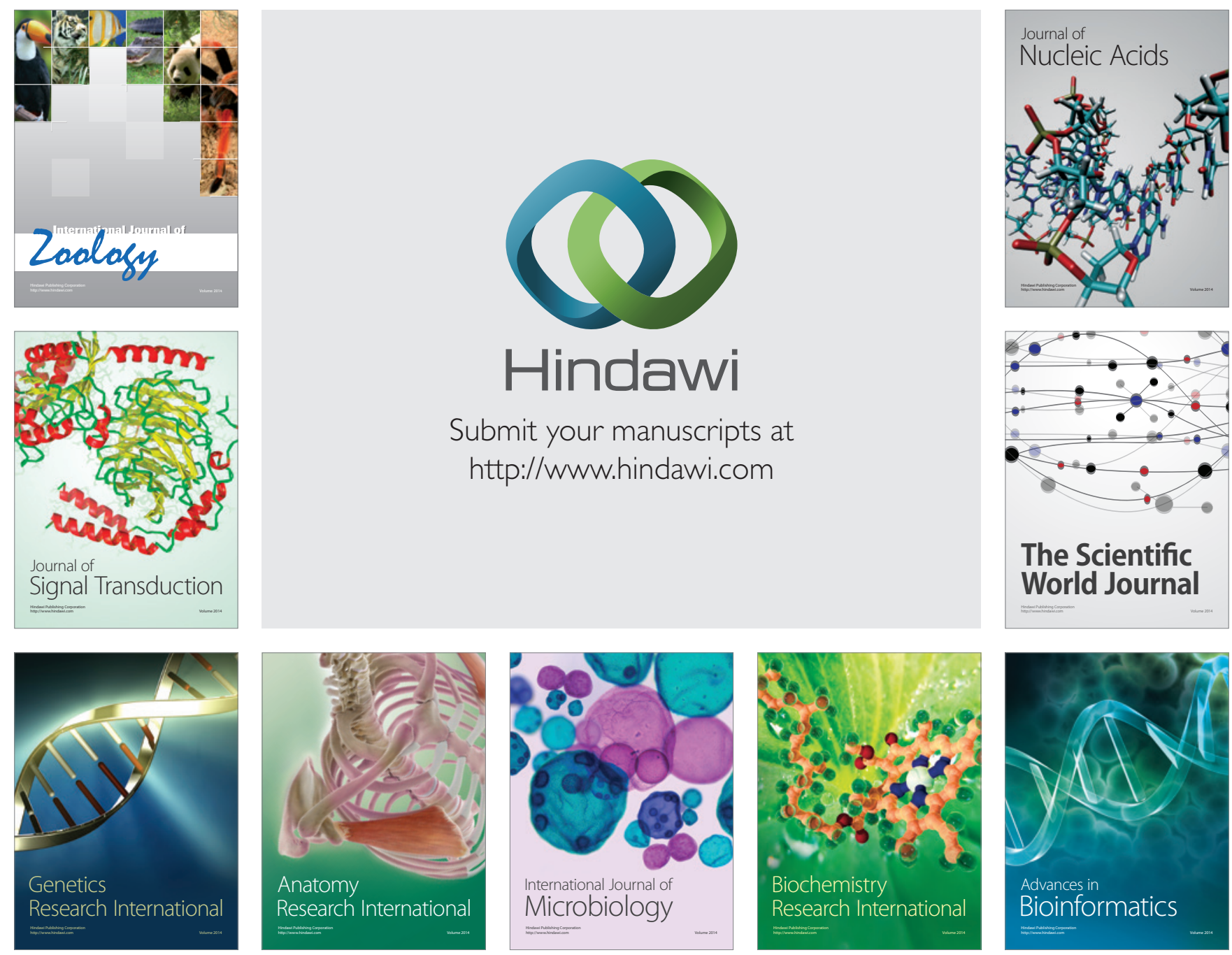

The Scientific World Journal
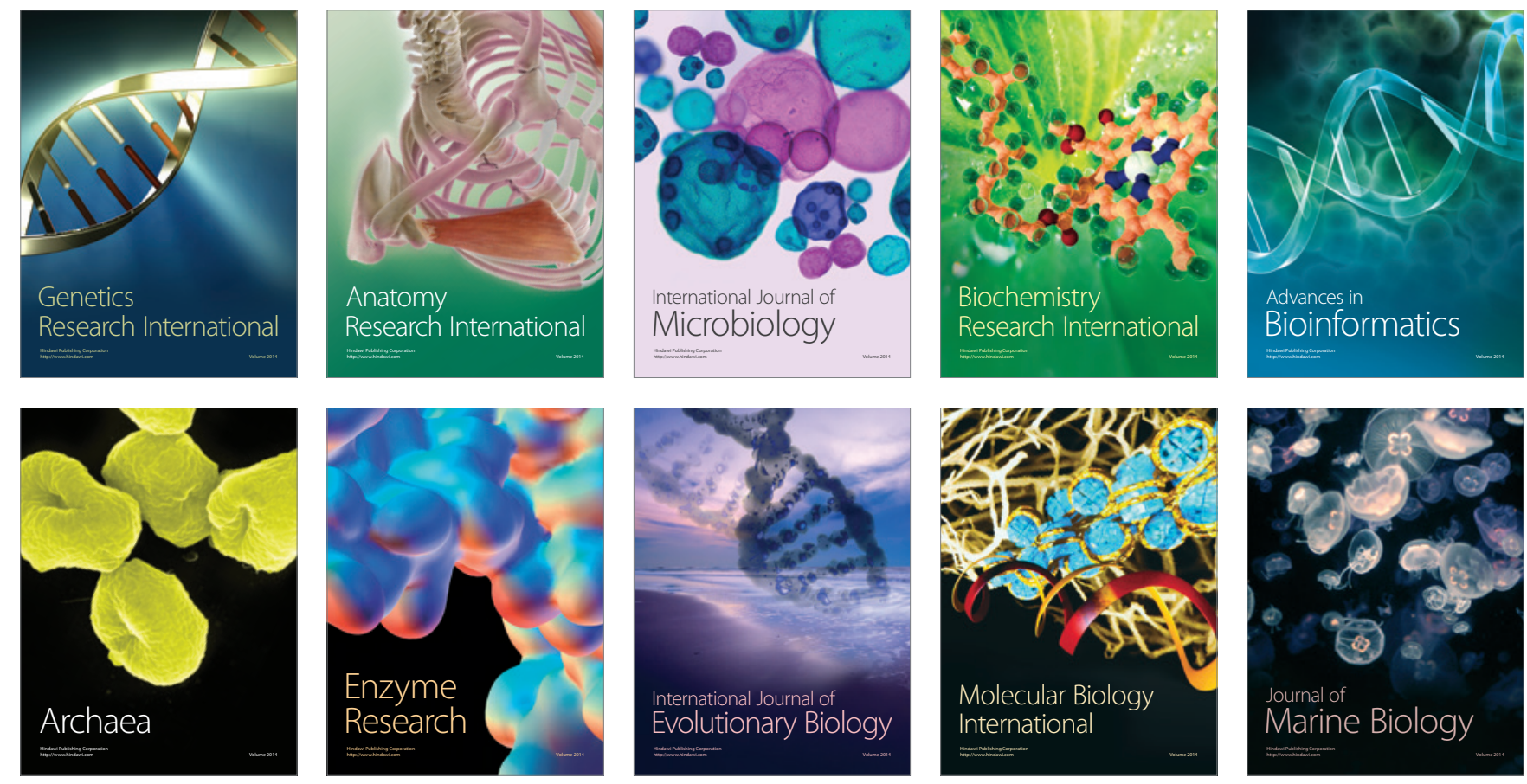